\section{Cureus}

Received 06/11/2018

Review began 06/18/2018

Review ended 08/23/2018

Published 09/10/2018

\section{(c) Copyright 2018}

Popov et al. This is an open access article distributed under the terms of the Creative Commons Attribution License CC-BY 3.0., which permits unrestricted use, distribution, and reproduction in any medium, provided the original author and source are credited.

\title{
Quantitative Analysis of Tumor-associated Tissue Eosinophilia in Recurring Bladder Cancer
}

\author{
Hristo Popov ${ }^{1}$, Ivan S. Donev ${ }^{2}$, Peter Ghenev ${ }^{3}$ \\ 1. General and Clinical Pathology, Forensic Medicine and Deontology, Medical University, Varna, BGR 2. \\ Clinic of Oncology, St. Marina University Hospital Varna, Varna, BGR 3. General and Clinical Pathology, \\ Forensic Medicine and Deontology, Faculty of Medicine, Varna, BGR
}

Corresponding author: Hristo Popov, hristo.popov@mu-varna.bg

\section{Abstract}

The relatively high incidence of recurrence of bladder cancer is a serious problem in clinical practice. At present, there are no objective microscopic criteria for evaluation of the tendency for local relapse. Besides the phenotypic properties of the tumor parenchymal cells, possible signs in regard to recurrence could also be derived from the peculiarities of the tumor stroma. The stromal reaction, manifested by inflammatory infiltration in the tumor is considered to influence the biological behavior of tumors. Also, a relationship has been reported between the number of eosinophils and the survival of patients. The aim of the present study is to analyze tumor-associated tissue eosinophilia (TATE) and to compare TATE density in the initial foci of age and gender-matched 156 cases of recurrent and non-recurrent bladder cancers; the tumors that have relapsed within six months after removal and contained statistically significant greater numbers of eosinophils in primary cancer sites. These results suggest that TATE may be one of the probable prognostic signs for local relapse of urothelial cancer.

Categories: Pathology, Urology, Oncology

Keywords: bladder cancer, recurrence, stroma, eosinophils

\section{Introduction}

Bladder cancer is a common urologic malignancy and has one of the highest recurrence rates [1]. Increasingly high morbidity rates impose a search for more relevant prognostic and predictive markers for tumors in stage pTa and pT1. Histologically, there are no convincingly objective criteria for assessing the tendency of local recurrence. In this regard, possible signs may be derived from some stromal characteristics, such as the presence of eosinophilic leukocytes, mast cells, lymphocytes, plasma cells, fibroblasts and myoepithelial cells. The classical understanding of the role of eosinophilic leucocytes is associated with allergic reactions and certain types of infections [2]. The presence of eosinophils in cancer has been established in some locations and malignant classes, despite some contradictory reports [3-5]. Tumor-associated tissue eosinophilia (TATE) may be associated either with good or poor patient prognosis [6-7].

Some studies support the opinion that eosinophils may have a role in tumor initiation [7]. In colorectal cancer, hematologic tumors, gastric cancer, lung cancer, gynecologic malignancies, and breast cancer, quantitative assessment of eosinophils in tumor tissue is proposed as one of the possible aspects of the histological evaluation, correlating to the patient prognosis $[3,5,8$ $18]$. 
The aim of the present study is to evaluate TATE in primary foci of recurrent and non-recurrent urothelial carcinoma of the urinary bladder.

\section{Materials And Methods}

A total of 156 cases of urothelial carcinoma were studied on the primary site formalin-fixed parathion embedded tissue sections, stained with hematoxylin and eosin (H\&E). Out of them, 78 had a disease recurrence within a period of six months. Their morphological features were compared to a control group of age and sex-matched 78 cases without recurrence for the same period of time. We examined the density of eosinophilic leukocytes per square millimeter of tissue surface.

We performed morphometric analysis by using digitally scanned slides via Leica Aperio ScanScope AT2 device (Aperio Technologies, Vista, CA) visualized on ImageScope V12.1.0.5029 (Aperio Technologies, Vista, CA).

Statistical analysis was carried out with SPSS Statistics v.23 using descriptive statistics. Categorical features were summarized with frequencies and percentages. The Mann-Whitney U test and Pearson correlation were used for comparison and estimation of correlations between the density of eosinophilic leukocytes in the stroma of non-recurrent and recurrent bladder cancer. Specificity and sensitivity of density of eosinophilic leukocytes in stroma for distinguishing recurrent disease patients (RP) from non-recurrent disease patients (NP) at three years of follow-up were evaluated with receiver operating curve (ROC) analysis. Diagnostic accuracy of biomarkers was also determined by obtaining the largest possible area under the curve (AUC) in ROC analysis. Kaplan-Meier survival curves and the log-rank test were used to compare the survival differences between groups. Although our study was not powered enough to compare different subgroups, hazard ratios (HRs) and corresponding $95 \%$ confidence intervals (CIs) were calculated by Cox regression models. Two-tailed p-values $(<0.001)$ were considered as significant.

\section{Results}

In the present study, eosinophilic leukocytes were present in the stroma of 66 tumors (42.3\%), either as scattered single cells, small groups of cells, or larger clusters. The Mann-Whitney test showed that there are significant differences in the number of eosinophils in the RP and NP group. TATE varied from entirely lacking eosinophilic leukocytes to moderately present and to very high density (Figures 1-3). 


\section{Cureus}



FIGURE 1: Case number 2: a 70-year-old male with high-grade urothelial carcinoma

The average number of eosinophils per square millimeter, in this case, is zero. Hematoxylin and eosin, original magnification $\mathrm{x} 200$.

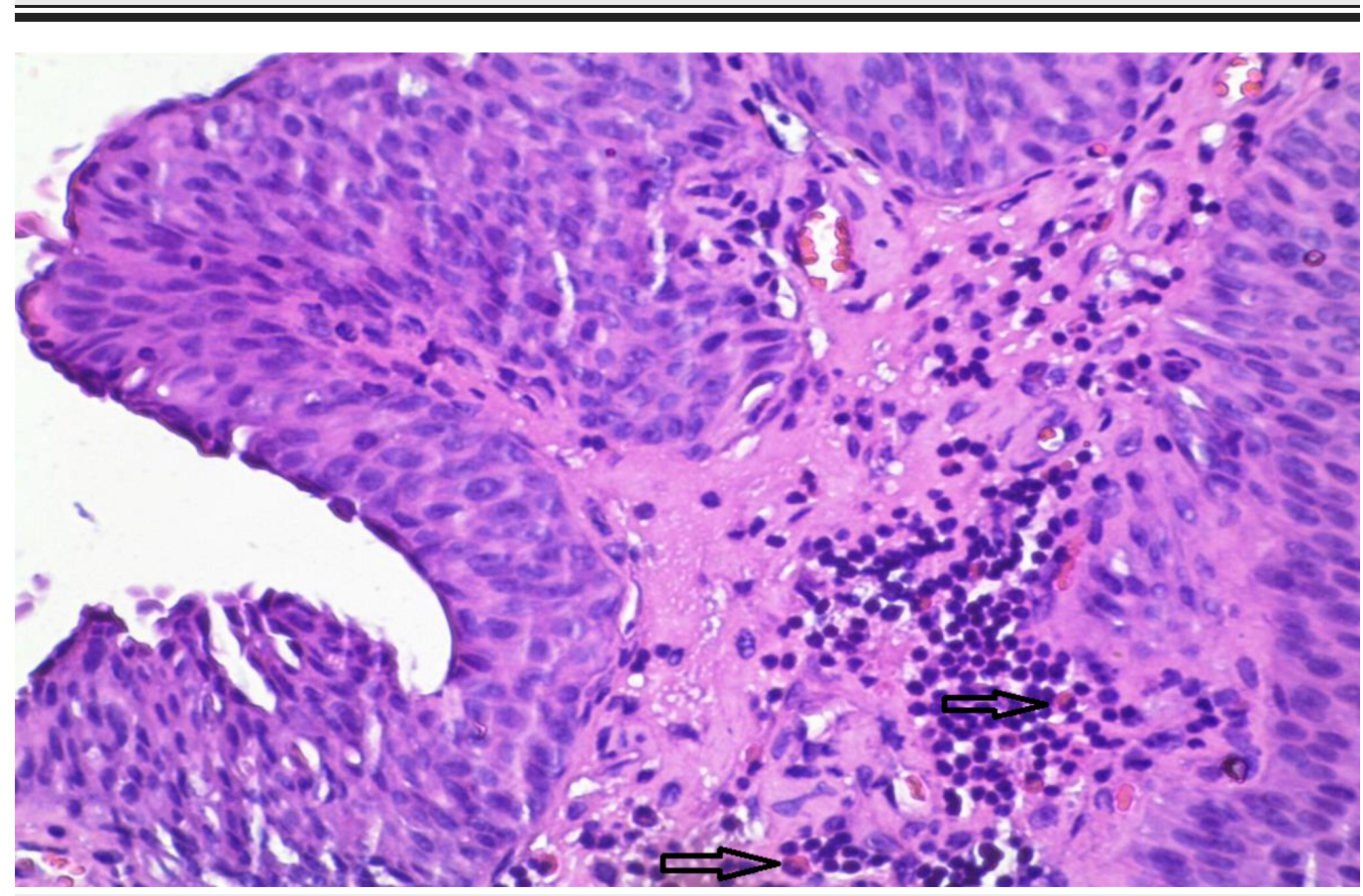

FIGURE 2: Case number 21: a 38-year-old male with high-grade urothelial carcinoma

The average number of eosinophils (arrows) per square millimeter, in this case, is 8.93.

Hematoxylin and eosin, original magnification x200. 


\section{Cureus}



FIGURE 3: Case number 15: a 67-year-old male with high-grade urothelial carcinoma

The average number of eosinophils (arrows) per square millimeter, in this case, is 35.21. Hematoxylin and eosin, original magnification x200.

The density of eosinophilic leukocytes in the tumor stroma varied severely between the RP and NP groups (AUC $=0.853,95 \%$ CI: $0.783-0.924, p<0.001$ ). At the optimal cutoff values of number of eosinophils, the sensitivity was $75.4 \%$ and specificity was $78.9 \%$ (Figure 4 ). 


\section{Cureus}



FIGURE 4: Receiver operating curve (ROC); $A U C=0.853,95 \%$ Cl: 0.783-0.924, $p<0.001$

AUC: area under curve; $\mathrm{Cl}$ : coefficient interval

Disease-free survival (DFS) was calculated as the time from the date of surgery to the date of documented recurrence of the disease. Cancer patients with a high number of eosinophils had no significant differences in DFS when compared with those with a low number of eosinophils (Figure 5). 


\section{Cureus}



FIGURE 5: Kaplan-Meier curve: no correlation between the number of eosinophils and disease-free survival (DFS)

The quantitative assessment of TATE in the tumor stroma shows statistically significant higher TATE in primary urothelial cancer, with a tendency to recur.

\section{Discussion}

The present study was designed to evaluate the significance of TATE in the stroma of urothelial cancer. We focused on one of the most peculiar features - the relatively high incidence of local progression as recurrence after surgical removal. The quantitative assessment of TATE in the tumor stroma shows the statistically significant higher density of eosinophils in primary urothelial cancers tending to recur and no influence on DFS. In a similar study of colorectal cancer (in squamous cell cancer with different location), high grades of TATE were associated with the lesser tendency of tumor metastasis.

Increased number of eosinophils may result from either an enhanced recruitment mediated by chemotactic mediators or a decreased apoptosis of eosinophils in the local tissue or both. The exact role of eosinophils in carcinogenesis - tumor transformation and/or progression is not quite clear.

Mature human eosinophils contain numerous highly basic and cytotoxic granule proteins, such as major basic protein, eosinophil peroxidase, eosinophil cationic protein, and eosinophilderived neurotoxin. Their activity is related to the release of these substances and basement 
membrane damage by eosinophil-derived cation proteins and peroxidase [9,19-24]. These products interfere in a variety of cellular processes involved in tissue remodeling and tumor behavior [9]. The presence of eosinophils in different amounts within the stroma of 66 bladder cancers (42.3\%) is obviously due to the action of potent eosinophil chemoattractants, such as eotaxin, platelet activating factor, C5a, interleukin-5, and immunoglobulin E (IgE) [12-13,18]. Some of these have been found in cancer patients - either in the tumor tissue [1] or in the blood [4]. Results from studies in other cancer locations suspect procancer effects of eosinophils via activation on stromal fibroblasts and angiogenesis [6,20]. In addition, eosinophils are capable to contact directly with tumor cells and trigger them into the apoptotic pathway. Collectively, TATE has been reported to be associated with a favorable prognosis for patients with esophageal squamous cell carcinoma and prostate carcinoma. Evidence derived from experimental models [19] also suggests the antitumor potential of TATE. However, up to date, the prognostic role of TATE in cancer patients remains conflicting rather than conclusive, given the inconsistent results from cancer of different origins

To the best of our knowledge, neither of these cellular interactions have been thoroughly studied in bladder cancer. Although most of the studies in other cancer location suggest a beneficial effect of TATE, the present results reveal reliable data for association of eosinophils in local recurrence of urothelial bladder cancer.

Keeping in mind the different significance of TATE, in regard to their location - both intraversus peritumoral and in different primary tumors, the most probable explanation seems to be that besides their direct interaction with tumor parenchymal and stromal cells, eosinophils have the potential to modulate the functions of other immune cells. Nevertheless, there is still a long way to understand the exact mechanism of the effect of eosinophils in cancer.

At least in urothelial bladder cancer, it is tempting to suggest that TATE might be regarded as a possible prognostic factor for local recurrence. So far, the evaluation of eosinophils represents a promising and readily assessable tool, and should therefore routinely be commented on in the pathology report.

\section{Conclusions}

The quantitative assessment of TATE in tumor stroma shows statistically significant higher TATE in primary urothelial carcinomas tending to recur. Although these findings need further explanation, it is tempting to suggest that TATE might be regarded as a possible prognostic factor for the local recurrence of bladder cancer.

\section{Additional Information}

\section{Disclosures}

Human subjects: All authors have confirmed that this study did not involve human participants or tissue. Animal subjects: All authors have confirmed that this study did not involve animal subjects or tissue. Conflicts of interest: In compliance with the ICMJE uniform disclosure form, all authors declare the following: Payment/services info: All authors have declared that no financial support was received from any organization for the submitted work. Financial relationships: All authors have declared that they have no financial relationships at present or within the previous three years with any organizations that might have an interest in the submitted work. Other relationships: All authors have declared that there are no other relationships or activities that could appear to have influenced the submitted work.

\section{References}

1. Sanli O, Dobruch J, Knowles MA, et al.: Bladder cancer. Nat Rev Dis Prim. 2017, 3:17022. 


\subsection{8/nrdp.2017.22}

2. Filipović M, Cekić S: The role of eosinophils in asthma . Med Biol. 2001, 8:6-10.

3. Pretlow TP, Boohaker EA, Pitis AM, et al.: Heterogeneity and subcompartmentalization in the distribution of eosinophils in human colonic carcinomas. Am J Pathol. 1984, 116:207-13.

4. Pereira MC, Oliveira DT, Kowalski LP: The role of eosinophils and eosinophil cationic protein in oral cancer: a review. Arch Oral Biol. 2011, 56:353-8. 10.1016/j.archoralbio.2010.10.015

5. Dalal BI, Das KC, Dutta TK, Malakar K: Local and systemic eosinophilia in patients with carcinoma of the uterine cervix undergoing radiation therapy: correlation with radiation response. Clin Oncol. 1992, 4:18-21. 10.1016/S0936-6555(05)80766-6

6. Ono Y, Ozawa M, Tamura Y, et al.: Tumor-associated tissue eosinophilia of penile cancer . Int J Urol. 2002, 9:82-7. 10.1046/j.1442-2042.2002.00424.x

7. Wong DT, Bowen SM, Elovic A, Gallagher GT, Weller PF: Eosinophil ablation and tumor development. Oral Oncol. 1999, 35:496-501. 10.1016/S1368-8375(99)00023-8

8. McGinnis MC, Bradley EL, Pretlow TP, et al.: Correlation of stromal cells by morphometric analysis with metastatic behavior of human colonic carcinoma. Cancer Res. 1989, 49:5989-93.

9. Pretlow TP, Keith EF, Cryar AK, et al.: Eosinophil infiltration of human colonic carcinomas as a prognostic indicator. Cancer Res. 1983, 43:2997-3000.

10. Samoszuk M: IgE in Reed-Sternberg cells of Hodgkin's disease with eosinophilia . Blood. 1992, 79:1518-22.

11. Samoszuk M, Ramzi E, Cooper DL: Interleukin-5 mRNA in three T-cell lymphomas with eosinophilia. Am J Hematol. 1993, 42:402-4.

12. Samoszuk MK, Nathwani BN, Lukes RJ: Extensive deposition of eosinophil peroxidase in Hodgkin's and non-Hodgkin's lymphomas. Am J Pathol. 1986, 125:426-9.

13. Samoszuk M, Nansen L: Detection of interleukin-5 messenger RNA in Reed-Sternberg cells of Hodgkin's disease with eosinophilia. Blood. 1990, 75:13-6.

14. Caruso R, Giuffrè G, Inferrera C: Minute and small early gastric carcinoma with special reference to eosinophil infiltration. Histol Histopath. 1993, 8:8.

15. Slungaard A, Ascensao J, Zanjani E, Jacob HS: Pulmonary carcinoma with eosinophilia demonstration of a tumor-derived eosinophilopoietic factor. N Engl J Med. 1983, 309:778-81. 10.1056/NEJM198309293091307

16. Bethwaite PB, Holloway LJ, Yeong ML, Thornton A: Effect of tumour associated tissue eosinophilia on survival of women with stage IB carcinoma of the uterine cervix. J Clin Pathol. 1993, 46:1016-20. 10.1136/jcp.46.11.1016

17. Samoszuk M, Lin F, Rim P, Strathearn G: New marker for blood vessels in human ovarian and endometrial cancers. Clin Cancer Res. 1996, 2:1867-71.

18. Samoszuk MK, Nguyen V, Gluzman I, Pham JH: Occult deposition of eosinophil peroxidase in a subset of human breast carcinomas. Am J Pathol. 1996, 148:701-6.

19. Kuper H, Adami HO, Trichopoulos D: Infections as a major preventable cause of human cancer. J Intern Med. 2000, 248:171-83. 10.1046/j.1365-2796.2000.00742.x

20. Wahl LM, Kleinman HK: Tumor-associated macrophages as targets for cancer therapy . J Natl Cancer Inst. 1998, 92:1583-4. 10.1093/jnci/90.21.1583

21. Tepper RI, Coffman RL, Leder P: An eosinophil-dependent mechanism for the antitumor effect of interleukin-4. Science. 1992, 257:548-51. 10.1126/science.1636093

22. Popov H, Ghenev P: Comparative analysis of relapsing bladder urothelial cancer . Acta Morphol Anthr. 2012, 19:192-5.

23. Ghiabi M, Gallagher GT, Wong DTW: Eosinophils, tissue eosinophilia, and eosinophil-derived transforming growth factor a in hamster oral carcinogenesis. Cancer Res. 1992, 52:389-93.

24. Wong DT, Elovic A, Matossian K, et al.: Eosinophils from patients with blood eosinophilia express transforming growth factor beta 1. Blood. 1991, 78:2702-7. 